\title{
COVID-19 and lessons from multi-hazard early warning systems
}

\author{
David P. Rogers ${ }^{1}$, Linda Anderson-Berry ${ }^{2}$, Anna-Maria Bogdanova ${ }^{1}$, Gerald Fleming ${ }^{1}$, Habiba Gitay ${ }^{1}$, \\ Suranga Kahandawa ${ }^{1}$, Haleh Kootval ${ }^{1}$, Michael Staudinger ${ }^{3}$, Makoto Suwa ${ }^{1}$, Vladimir Tsirkunov ${ }^{1}$, and \\ Weibing Wang 4 \\ ${ }^{1}$ World Bank, Washington, DC, USA \\ ${ }^{2}$ College of Science and Engineering, James Cook University, Cairns, Australia \\ ${ }^{3}$ Zentralanstalt für Meteorologie und Geodynamik (ZAMG), Vienna, Austria \\ ${ }^{4}$ Weibing's is School of Public Health, Fudan University, Shanghai, China
}

Correspondence: David P. Rogers (drogers@bluewin.ch)

Received: 4 May 2020 - Revised: 3 June 2020 - Accepted: 15 June 2020 - Published: 13 July 2020

\begin{abstract}
Having a common framework for early action to cope with complex disasters can make it easier for authorities and other stakeholders, including populations at risk, to understand the full spectrum of secondary and tertiary effects and thus where to focus preparedness efforts, and how best to provide more targeted warnings and response services. Meteorological and hydrological services world-wide have developed and implemented MultiHazard Early Warning Systems (MHEWS) for weather and climate related hazards that are now being expanded and transitioned towards Multi-Hazard Impact-based Early Warning Systems (MHIEWS). While it is still early days it is becoming clear that there are useful lessons from this approach in the COVID-19 global pandemic, and some valuable insight to be gained in risk communication, risk analysis and monitoring methodologies and approaches. The ability to understand and respond effectively to warnings through appropriate behaviours and actions is central to resilient societies and communities. By avoiding physical, societal and economic harm to the greatest extent possible, recovery from a hazard is likely to be faster, less costly and more complete.

MHIEWS can be a common approach for all hazards and therefore more likely to become a trusted tool that everyone can understand and use as a basic element of their national disaster risk management system. The interconnectedness of hazards and their impacts is a strong motivator for a common approach. One of the lessons from the COVID-19 pandemic and extreme weather events is the need to understand the vulnerability of individuals, communities and societies so as to provide reliable, targeted guidance and warnings and the willingness and capacity to prepare for a reasonable worst-case scenario based on informed long-term planning. Meteorology and hydrology are making good progress in this direction and the process can be readily applied to health and other sectors.
\end{abstract}

\section{Introduction}

The COVID-19 crisis and extreme weather events provide important lessons in understanding risk and creating effective multi-hazard impact-based early warning systems (MHIEWS). In the past decade, meteorology has made significant progress in applying likelihood of occurrence and impacts to early warning systems. An important tenet of the probability versus impact matrix (Fig. 1), which is a commonly used qualitative assessment method, is being prepared for the very low probability high impact event. This leads to the related concept of the "reasonable worst case" for which good risk management would require us to be prepared. Examples of preparedness and anticipatory action would be ensuring economic safety nets are in place, the availability of personal protective equipment and access to ventilators to cope with complications of a pandemic; or the reinforcement of shelters to protect vulnerable people from the impact of storm surges or flooding caused by tropical cyclones; 


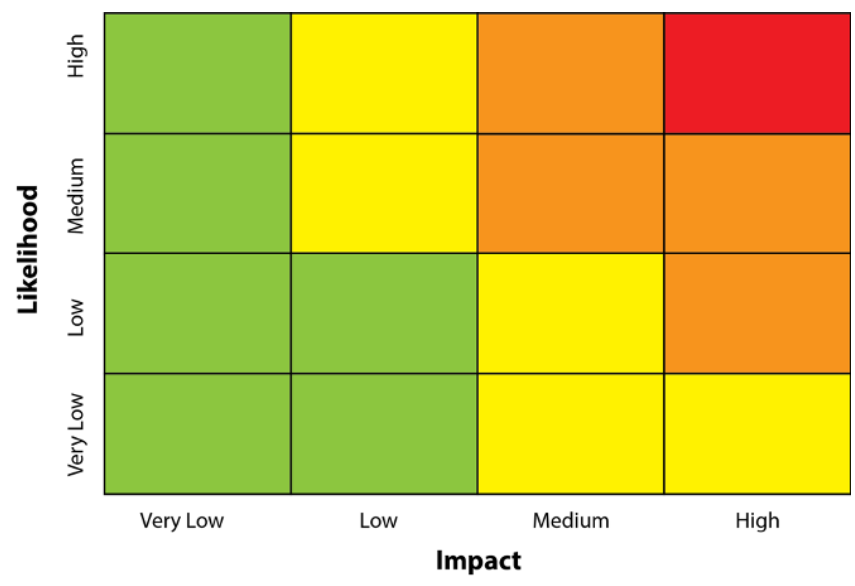

Figure 1. A probability versus Impact matrix for any hazard. The colours are assigned based on an assessment of the risk, which typically has four categories - very low $(<20 \%$ green), low $(20 \%-$ $40 \%$ yellow), medium (40\%-60\% orange) and high ( $>60 \%$ red). In practice, only the right half of the matrix is considered; i.e., when the impact is medium or above.

the cleaning of drainage systems to reduce flood impact on communities.

The probability versus impact risk matrix is used in the national risk assessments carried out by many countries and mandated by the EU for Member States ${ }^{1}$. The Joint Research Council of the European Commission has provided a publication with recommendations for national risk assessment and disaster risk management in the EU which incorporates a similar impact/probability matrix approach to mapping risks ${ }^{2}$. What is evident from both publications, however (and, presumably in national risk assessments from many other countries) is that the risks arising from different sources are largely treated independently, with little consideration of the potential societal and economic consequences as one hazard begets another, or of multiple independent hazards happening at the same time. In the world of meteorology, however, the move towards Impact-Based Forecast and Warning Services (WMO, 2015) has prompted an explicit examination of the inter-relationships between natural hazards, vulnerability and risk in thinking through the possible consequences of a severe weather event. Typically, the recovery and rebuilding efforts in many island nations, for example, are 3-5 years or even longer if the areas are remote. It is worth noting that some of these events (e.g. Hurricane Maria in Puerto Rico, or any drought event) have permanent impacts on societies, which are least resilient (Rogers et al.,

\footnotetext{
${ }^{1}$ see for example the National Risk Assessment for Ireland available at https://www.gov.ie/en/publication/ 709bf3-a-national-risk-assessment-for-ireland-2017/, last access: 26 June 2020

${ }^{2}$ see https://ec.europa.eu/jrc/en/publication/recommendationsnational-risk-assessment-disaster, last access: 26 June 2020
}

2018). In many countries, similar impacts may be anticipated from COVID-19.

In the case of each potential hazard, a simple theoretical analysis of rational decision-making suggests that proactive action should be taken if the forecast probability of a lifethreatening event is greater than the ratio of the costs of protection (e.g., infrastructure investment) and the losses when no protective measures are taken and an event occurs (e.g., loss of life, damages, economic disruption) (Murphy, 1969; Coughlan de Perez et al., 2015; Palmer, 2019). The COVID19 pandemic implies in hindsight that the costs of mitigation were very small compared with the losses and therefore more should have and could have been done to mitigate the risk (Harford, 2020); however, optimism bias or our tendency to underestimate the impact of the highly improbable (Kahneman, 2011; Taleb, 2010) compounds our inability to be prepared. They also illustrate the challenges in anticipating the wider societal implications of natural hazards, the complications in estimating the potential impacts and costs, and throw into sharp focus the value judgements that must be made in balancing protection costs against human and economic impact.

Early detection, early warning and early action are also important precepts as ex ante mitigative actions cannot completely eliminate risks. Early detection and early warning enable early action, but the latter will only occur if there is a clear understanding of the potential impact that is based on rigorous risk assessment and includes the range of environmental and societal consequences and complete understanding and acceptance of the need to act on high impact events, even if the probability of occurrence is very low. The actions may differ depending on the likelihood, but the impact will be the same if the event occurs. In practice, we should be ready to undertake preparatory actions for all medium and high impact events. The definition of high, medium and low impact is hazard specific and determined by collecting impact data and through expert opinion and analysis.

Meteorology has developed the capacity, largely through ensemble forecasting methods, to apply probabilistic forecasting techniques to its warning systems and it has sophisticated tools to ensure the reliability of the system (Palmer, 2019). Similar approaches are also being applied to epidemic forecasts using multi model ensemble techniques, which produce broader and more realistic possible trajectories of epidemics (Chowell et al., 2020). Probabilistic forecasting of complex cascading catastrophes remains a challenge, but one that must be addressed. We are still vexed with the problem of forecasters not having sufficient confidence to issue guidance or warnings for low likelihood events, even when the potential impact is high. Some of this is down to the "boy who cried wolf" syndrome, but some is also down to the limited ability of humans (and all forecasters are human!) to imagine an event completely outside their own experience, and thus to warn for that event. 
Ironically, many users are much more likely to want and use probabilistic information because most will base their decisions on their understanding of their own costs and potential losses. For example, farmers in Bangladesh, risking loss of livelihoods, do take action on a very low or low probability of a high impact event to avoid or mitigate losses (Webster et al., 2010). This could take the form of moving livestock to higher ground or by taking out an insurance policy. In the first case responding to the warning and in the second in response to the risk analysis. Providing cash to households based on forecasts of extreme events is another approach (Gros et al., 2019). The different response of countries to the COVID19 pandemic, especially those having difficulties managing caseloads, suggests that a more rigorous approach to understanding the full spectrum of risk is needed.

This inability to accept low probability high impact situations must be overcome if the benefits of MHIEWS are to be realised and high adverse impacts avoided or mitigated. Reliably identifying a low probability, high impact flood hazard 5 to $7 \mathrm{~d}$ ahead, for example, would allow civil protection to prepare resources in this time frame professionally without stress. If their reaction is only on high probability forecasts, making resources available in the necessarily shorter timeframe is much more stressful and, in most cases, also costlier. From the public perspective, high probability forecasts have a greater chance of being accepted and reacted upon; thereby building more trust towards authorities and the MHIEWS. Taking COVID-19, as an example, the low probability time frame in Central Europe concerned politicians as the decision-makers, who could have saved time and money gathering and improving response capacity. The high probability scenario started around early March where the focus should have been on public behaviour, accepting hygienic measures, lockdown and social distancing. The scramble in many countries to acquire personnel protective equipment during the high probability phase of the hazard highlights the failure to act on the low probability scenario and the dire consequences for frontline workers.

These basic concepts must be kept in mind as we explore MHIEWS in more detail. Decision making for low probability and high impact events is often hindered by the fact that government decision-makers are focused day-to-day on solving high probability, relatively minor, but media relevant, problems that divert attention from the potential catastrophic impact of low likelihood events.

\section{Multi hazard impact-based early warning systems and services}

During the past decade considerable attention has been focused on developing, implementing and improving multi hazard early warnings systems and, in particular, in the context of meteorological forecasts and warnings, extending this concept to include impact-based forecast and warning ser- vices (Tang et al., 2012; WMO, 2015) and extending the concept to specific sectors including health (Ghebreyesus et al., 2008). A common methodology has evolved, which ultimately aims to provide guidance to people-at-risk and those responsible for mitigating those risks in a form that is understandable and actionable. MHIEWS are designed to support the early alerting of governmental and nongovernmental decision-makers permitting preparatory steps to be taken ahead of issuing public warnings. This requires combining information about specific hazards with the likely impacts of those hazards on people, livelihoods and property. It is also essential to understand the cascading effect of hazards where a single natural event may have a multiplying effect resulting in primary, secondary and tertiary hazards. For example, a meteorological event such as a tropical cyclone produces heavy rainfall, which in turn causes flooding disrupting transportation networks, energy supplies and other critical infrastructure, loss of life or physical harm, social isolation, interruption to employment and livelihood activities, and psychological distress. COVID-19 follows the same pattern resulting in major social and economic hazards and consequential impacts.

Tang et al. (2012) stress the importance and effectiveness of a multi hazard approach to disaster reduction by understanding how hazards can produce a series of social consequences, which are also public hazards. The emphasis on impacts, therefore, also implies that warnings should be related to multiple hazards since the initial event can cause a series of cascading threats or consequential effects - public health, accidents, infrastructure damage, civil unrest, food insecurity, etc. Ideally, each of these should also be considered and the means to predict their likelihood developed, so that a more complete modelling of impacts can be achieved. Unfortunately, despite the demonstrated usefulness of the concept in meteorology, and best efforts to date (IFRC, 2012; WMO, 2019), multi hazard impact-based early warning systems have remained primarily focused on hazards of meteorological origin. A renewed effort is needed to make MHIEWS fully multi-sectorial, inclusive of biological hazards, regardless of the cause of the underlying events (Yao et al., 2020b). This is an important part of a more effective and streamlined disaster risk management.

In all MHIEWS, the ultimate aim is to provide people with information that they understand, trust and act on. The pandemic crisis highlights the importance of trusted sources of information (Hua and Shaw, 2020). This suggests the benefits that would flow from a common framework for all warning services, which emphasizes the impact of the hazard rather than the technical jargon that normally accompanies the description of the hazard itself. In meteorology, the emphasis is shifting from "what the weather will be to what the weather will $d o$ " and this can be generalized to any natural, socioeconomic or technological hazard by focusing on the impact of the hazard. This has come about because, despite huge technological advances in forecasting meteorological and hy- 
drological hazards, we continue to suffer major human and economic losses through incomplete awareness and understanding of the potential breadth and depth of the impacts of those hazards. Similarly, although COVID-19 has unique epidemiological aspects, it is a known class of virus and the behaviour of a pandemic can be modelled, with advice, guidance and warnings issued based on detection (observation) or model forecasts (e.g., Chowell et al., 2020). However, the actions taken by many governments suggest any or all of the following: warnings have not been heeded; the risks poorly understood and discounted; there are no funding mechanisms to take early action; capacity is lacking to do the things that need to be done, even if the money is available; long term planning is lacking to make best use of the warning. Unfortunately, this is a common theme for many hazards and a major obstacle in achieving the goals of the Sendai Framework for disaster risk reduction (United Nations, 2015). The response to hydrometeorological hazards provides some insight and, through generalizing MHIEWS concepts, we may be able to identify a way forward.

Each year the impacts of severe hydro-meteorological events around the world give rise to multiple casualties and significant damage to property and infrastructure, with adverse social and economic consequence for communities that can persist for many years. All this happens in spite of the fact that many of these severe events are recurrent, have been well forecasted, with accurate warning information disseminated in a timely fashion by the responsible authority.

The reasons for this apparent disconnect and the failure of this information being fully utilised to support effective defensive action and mitigation decisions lays in the gap between forecasts and warnings of the hazardous events and an understanding of the risk associated with their potential impacts, both by the authorities responsible for civil protection/emergency management and by the population at large. The forecast and warnings information should be disseminated in a range of formats and styles and across as many communication platforms as are appropriate to the recipient audience. The content and context of the information needs to be believed and trusted as coming from an expert skilled and non-biased source and, when it is confirmed with secondary sources, it should be consistent in its message. It is important that the recipient at-risk is prepared to accept and enabled to act on this information (Anderson-Berry et al., 2018).

If the gap between forecast and warnings information and effective loss minimising actions is to be closed, then an allencompassing approach is needed to observe, detect, model and predict severe events and the consequent cascade of hazards through to impacts. Tackling this problem requires a multi-disciplinary approach to identify, understand, assess and address risks. This requires access to the best possible physical, social and behavioural scientific and humanitarian sector understanding, and the optimum services, to manage multi-hazard events. This approach will provide the best pos- sible evidence base on which to make the costly decisions on infrastructure and other preventative investments to protect the population in the future.

All countries should provide their citizens and economic sectors with actionable information that, wherever possible, identifies the timing and anticipated impacts of specific hazards. An informed population that fully understands what a hazard will do is more likely to engage in appropriate behaviour and take the necessary actions that protect their lives and livelihoods ${ }^{3}$. Clarity and trust in communication is essential, especially when the likelihood is very low or low, but the potential impact is very high.

In the case of meteorological hazards, the meteorological and hydrological services must work closely with emergency services, disaster reduction and civil protection agencies to share data and to interpret forecasts into a form that results in early warning and early actions by everyone (Rogers and Tsirkunov, 2013; Rogers et al., 2019). It is important that local authorities and agencies with an understanding of community dynamics, physical and societal infrastructure and social networks are included in this working partnership. They will hold extensive knowledge of the vulnerability of individuals and community sectors, of both formal and informal communication networks and have the ability to engage multiple stakeholders. They will also have valuable insight around and the likely behaviour of people during an emergency. This is a new area for many meteorological and hydrological forecast and warning services, since it requires extensive knowledge of how meteorology and hydrology affect day-to-day activities. None of this knowledge and awareness may be available to the weather service providers in developing countries, some of which already struggle to produce basic meteorological and hydrological forecasts and services. Coproduction of these new warning services involving all key stakeholders is required ${ }^{4}$.

The same issues apply to health related and technological hazards and therefore all of society would benefit from a common approach to the development of multi hazard early warning and response systems. Given that pandemics, tropical cyclones, tsunamis, heat and cold waves and droughts may affect many countries simultaneously, and also that the cascading impacts of a hazard even in a geographically confined area can have global consequences, as was the case with the 2011 flood in Thailand for the global supply chain for hard disk drives, international cooperation at the highest levels of government and industry is an important element in ensuring that tools are available and responses appropriate, as evinced by the COVID-19 pandemic (Kluge, 2020). The disaster management laws of many countries cover epidemics or

\footnotetext{
${ }^{3}$ https://public.wmo.int/en/media/news/ cyclone-amphan-highlights-value-of-multi-hazard-early-warnings, last access: 26 June 2020

${ }^{4}$ https://manual.forecast-based-financing.org/chapter/ set-the-trigger/?pdf=chapter, last access: 26 June 2020
} 
pandemics as disasters; therefore, integrating epidemics and pandemics in a MHIEWS is a rational approach to improve coordination, forecasting, warning and response. Samoa, as an example, did this for the measles outbreak that affected the country in December 2019 which in turn also enabled them to take early action as COVID-19 started spreading into the Pacific.

\section{Basic elements of a MHIEWS}

Given that the goal is to provide guidance to people-at-risk and to those responsible for mitigating those risks in a form that is understandable and actionable (IFRC, 2012), the requirement is to combine information about specific hazards with the likely impact of those hazards on people, their livelihoods and property. Depending on the knowledge of the hazard, and the vulnerability and exposure of people, the approach may be either qualitative, depending on expert knowledge, or quantitative, depending on measurement.

A MHIEWS has five basic steps: (1) A common framework for visualizing warnings; (2) Hazard identification; (3) vulnerability assessment; (4) risk matrices; and (5) advisory and alerting. These are described in detail below.

\subsection{A common framework for visualizing warnings}

Mapping the distribution of hazards and impacts geographically is the preferred approach using administrative boundaries as polygons to present the location of the hazard and impact. Each of these polygons or grid cells may have its own granular structure depicting a much finer mesh and facilitating more detailed warning information. The advantage of mapping administrative boundaries is the presence of public officials in each of the "grid cells", who have a responsibility for public safety. The apps developed in several Asian countries to trace people potentially exposed to COVID-19 lend themselves to this approach, albeit with data privacy issues, which must be resolved (Cho et al., 2020). A major obstacle that needs to be resolved is to balance privacy and the use of personal data in such systems with the overall public good which can be attained through their deployment. In any case it is important that individuals receive reliable information that they can trust and act on.

Meteoalarm, as an exemplar of a common framework for warning services in the sphere of hydro-meteorology, has extended the visualization of such warnings to all of Europe using consistent colours to represent the severity of the hazard, together with employing a standard set of symbols for each of the meteorological hazards - wind, rain lightning/thunderstorms, heat and snow/ice (Staudinger, 2008) which are displayed to show the type of hazard (Fig. 2). This approach could be readily extended to all hazards and could have been of significant benefit in visualizing the dynamic development of the COVID-19 pandemic across Europe. Plans exist to extend the concept of Meteoalarm be- yond Europe through the World Meteorological Organization's Global Multi-Hazard Alerting System (GMAS) and through World Bank investment projects aimed at transforming national meteorological and hydrological services, which include the use of the Meteoalarm concept and software.

Figure 3 shows the detailed warning system for Germany, which could be emulated in any country. More effort is needed to include a wider range of hazards and their impacts.

Ultimately the colour coding should be related to the impact of the hazard rather than the hazard itself, as depicted in the risk matrix in Fig. 1, reflecting the shift in emphasis to actionable information for those at risk. In Wuhan, a colour coded QR-code was used to inform the public on safety with green indicating safe, yellow indicating a need to be cautious, and red a "cannot enter" (Hua and Shaw, 2020), which largely follows an approach first introduced into China for meteorological warnings in Shanghai (Tang et al., 2012).

\subsection{Hazard identification}

The identification of all events impacting the territory of a country, and the primary and secondary hazards is ideally required. The primary hazards are caused directly by the event in nature and cannot be mitigated to any significant extent (e.g., rain will fall, a virus will exist). The secondary hazards are a consequence or impact of the primary hazard and can often be partially mitigated (e.g., structural works can reduce the possibility of a surface flood in an urban area, or hospital equipment can be stockpiled). The tertiary hazards may be caused by the primary and secondary phenomena or may be a consequence of human failure and will have substantial societal impacts. Tertiary hazards may also evolve from efforts to mitigate the primary (natural) and secondary hazards. The societal impacts have the greatest scope for mitigation by either structural or social measures to reduce exposure and vulnerability and build capacity and capability. In the case of secondary and tertiary hazards, the hazard and the impact of the hazard may be closely related and interconnected. Each of the hazards leads to further impacts, but not all impacts are associated with further natural hazards. For example, economic disruption can be caused by social behaviour, which spreads disease, and the consequence of the economic disruption can have a significant impact on productivity and public financing, which in turn is a hazard with long-term impacts on poverty and the well-being of society. Table 1 summarizes primary, secondary and tertiary hazards and associated high impacts for a virus, cyclone and earthquake. The list of impacts is illustrative and not exhaustive. Impacts are generally place specific and should be determined based on local knowledge.

Collecting hazard impact data is challenging and often recorded in a very general way; for example, the number of affected people, which not useful for creating impact-based warnings. 
Table 1. Example of Events, primary, secondary and tertiary hazards and associated impacts.

\begin{tabular}{llll}
\hline Event & Primary hazards & Secondary hazards & Tertiary hazards \\
\hline Virus & - Infectious disease & $\begin{array}{l}\text { - Disease spread by human be- } \\
\text { haviour } \\
\end{array}$ & - Overwhelming of health services \\
& - Economic disruption & - Loss of productivity \\
& & - Public finances overwhelmed \\
& & - Loss of education opportunities \\
& & - Unemployment \\
& & - Civil disobedience \\
& & - Psychological problems \\
\hline
\end{tabular}

High impacts:

- Excess number of deaths caused directly by virus and indirectly due to overwhelmed health services

- Civil disobedience resulting in increased rates of transmission of disease

- Severe disruption to global economy resulting in severe financial losses and economic recession

- More people in poverty

- Food insecurity especially in poorest communities increasing morbidity

- Increased crime related to loss of income and exploitation of crisis

- Civil unrest potentially destabilizing societies

- Restructuring of social priorities

- Increased international tensions resulting in conflict

\begin{tabular}{llll}
\hline Cyclone & - Strong wind & - River flood & $\begin{array}{l}\text { - Damage in Dams and appurtenant structures, } \\
\text { embankment, irrigation and drainage facilities, } \\
\text { pumping facilities }\end{array}$ \\
& & - Submerging paddy fields \\
- Lightning & - Surface water flooding & - Migration \\
- - Teavy rainfall & - Flash flood & - Loss of infrastructure systems and services \\
& - Landslides & (shelter, transportation, schools, hospitals, en- \\
& & ergy supply, communication) \\
& - Storm surge & - Waterborne diseases \\
& - Water level rise in reservoirs & - Environmental degradation \\
& - Riverbank erosion & - Snake bite \\
& - Muddle & - High sediment transport into reservoirs
\end{tabular}

High impacts:

- Loss of property and livelihoods resulting in increased poverty and homelessness

- Excess number of deaths and injuries due to event and subsequent disease outbreaks

- Loss of agricultural land and potable water resources

- Widespread food and water insecurity especially in poorest communities increasing morbidity

- Risk of theft of property results in people not taking shelter

- Civil unrest and political instability

- Severe disruption to transportation networks

- Widespread population displacement

\begin{tabular}{|c|c|c|c|}
\hline Earthquake & $\begin{array}{l}\text { - Shifting Geological } \\
\text { Formation }\end{array}$ & $\begin{array}{l}\text { - Building Collapse } \\
\text { - Road and Rail fracture } \\
\text { - Tsunami } \\
\text { - Fire } \\
\text { - Liquefaction }\end{array}$ & $\begin{array}{l}\text { - Damage in Dams and appurtenant structures, } \\
\text { embankment, irrigation and drainage facilities, } \\
\text { Pumping Facilities, } \\
\text { - Loss of Infrastructure System and Services } \\
\text { (shelter, transportation, schools, hospitals, en- } \\
\text { ergy supply, communication) } \\
\text { - Coastal Flood } \\
\text { - Changes in Ground water formation } \\
\text { - Psychological problems }\end{array}$ \\
\hline
\end{tabular}

High impacts:

- Excess number of deaths and injuries due to collapse of homes, buildings and other infrastructure

- Widespread financial losses

- Severe disruption to transportation networks

- Widespread population displacement

- Decrease in water storage capacity 


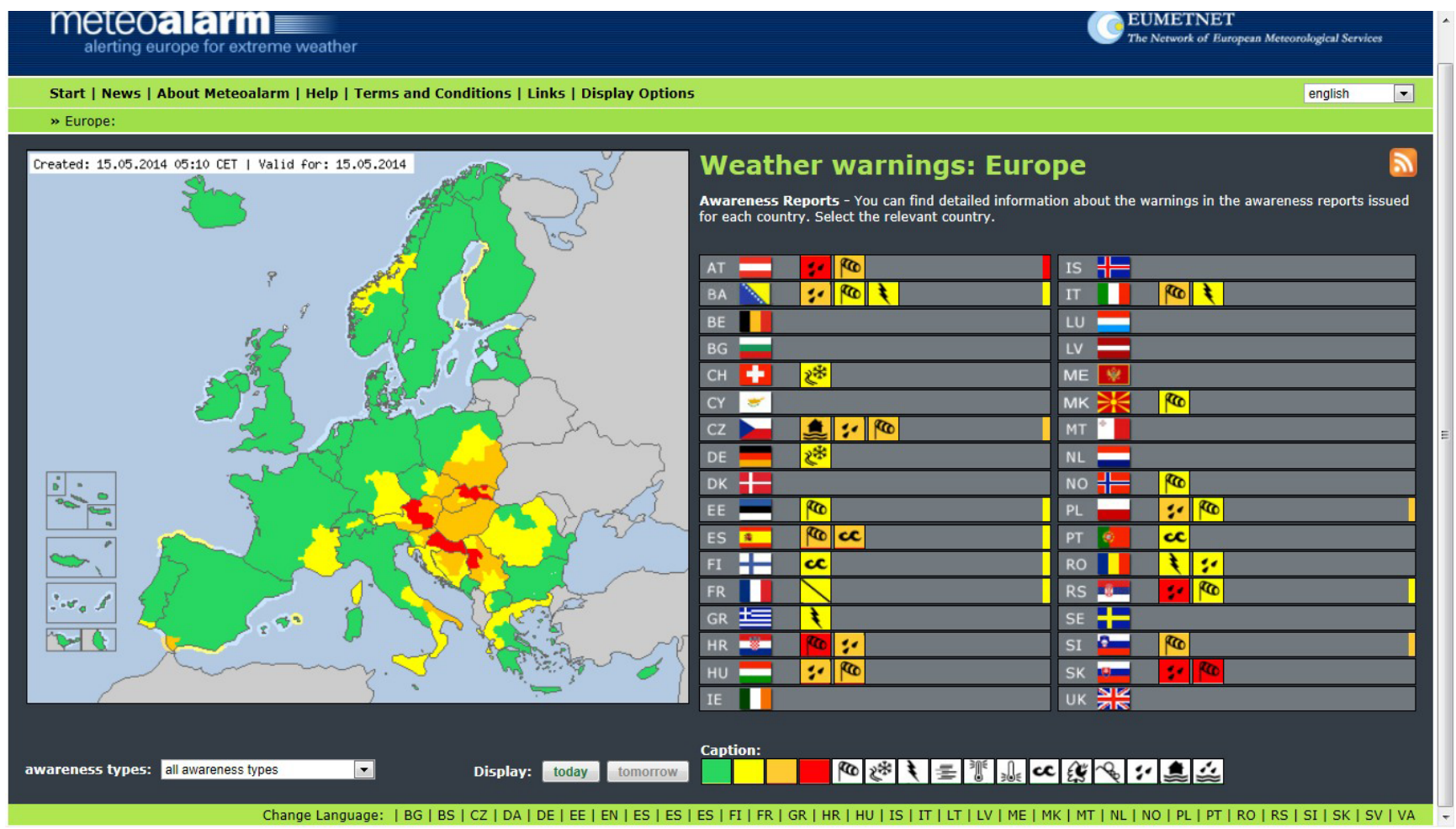

Figure 2. Example of the graphical output from the Meteoalarm portal. The colour coding is consistent across all participating European countries.

\subsection{Exposure and vulnerability assessments}

Understanding who is at risk depends on gathering information on vulnerability and exposure involves many different entities including social and behavioural scientists, disaster managers, non-governmental organizations, civil and structural engineers, risk finance and risk transfer specialists, and, of course, those at risk. These data are an important layer of information within any decision support system. The responsibility for compiling and updating this information often falls to disaster managers and non-governmental organisations, which work with mostly disadvantaged communities. However, it is rarely compiled in a single operational data base and is often incomplete and often not of very good quality. Social and behavioural scientists are critical to understanding how differently abled people access, comprehend and use warning and forecast information. Data needs to be collected at the level of the individual and updated regularly.

The vulnerability of infrastructure systems and services must also be quantified. For example, the vulnerability of bridges and roads to inundation or destruction due to flooding, or the likely requirements for specialised medical equipment, should be estimated. Understanding sectorial interdependencies is also necessary to determine vulnerabilities and therefore to develop the appropriate impact-based forecasts and warnings. Addressing these vulnerabilities is a way to increase resilience and reduce the risk of disaster stemming from a failure to cope adequately with the primary and secondary hazards (Rogers et al., 2018). Not doing so can result in persistent and sustained loss of economic capacity (Moteff, 2012). By understanding the vulnerability of the infrastructure system and services to the primary and secondary hazards, and the decisions that have resulted in mitigating actions - or not - it is possible to provide more accurate and timely impact-related warnings that would protect a population from existing weaknesses in infrastructure, which compound the threat of mortality and morbidity posed by the initial hazards. Collapse of buildings, bridges, and roadways, the loss of ICT, electricity, transportation, health services, employment and sanitation and the decision and opportunity for people to remove themselves from harm's way frequently contribute to creating, enhancing or diminishing the circumstances of subsequent disasters. Already available vulnerability and exposure information on hydromet hazards can be used for the analysis of epidemic risk, and the cascading impacts.

\subsection{Development of risk matrices}

Probability versus impact matrices are required for every hazard and each sector likely to be affected. It requires knowledge of the hazard and expert knowledge of the likely impact on a specific sector. This may or may not be informed by a formal vulnerability assessment. At its most basic it 


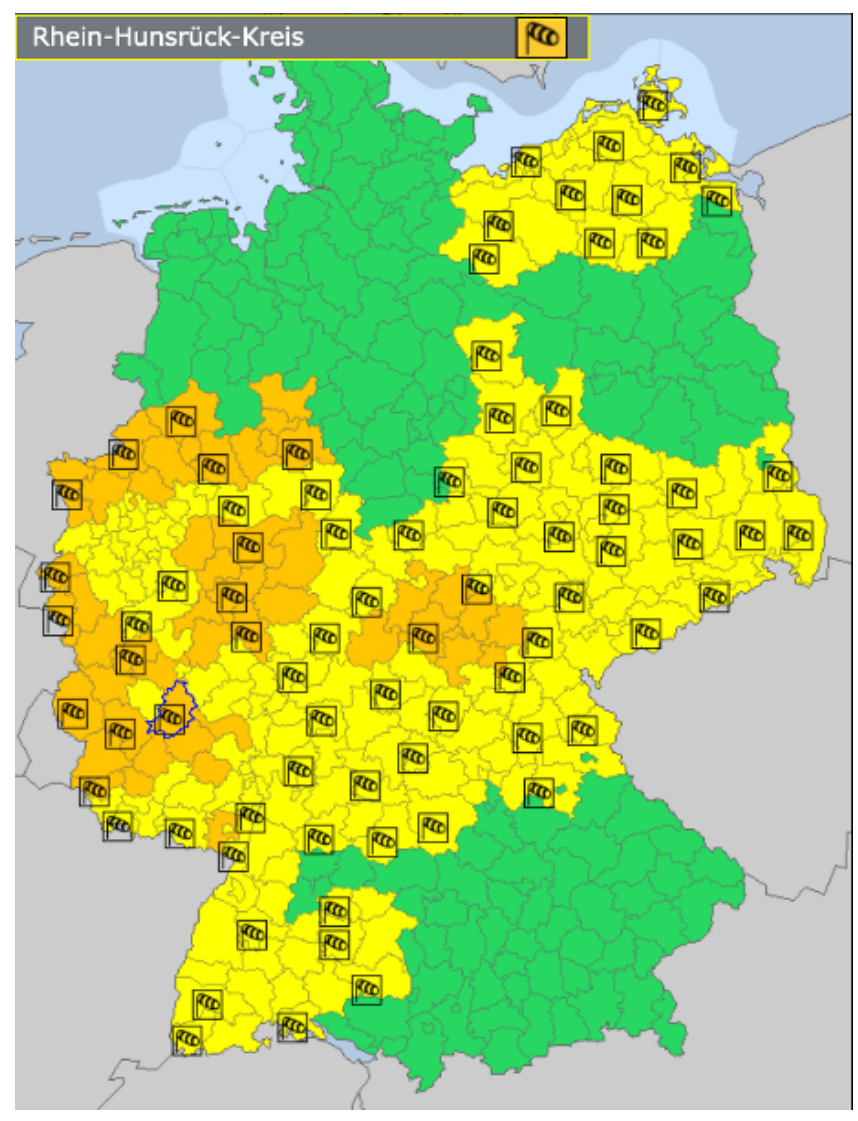

Figure 3. Example of the German Weather Service (Deutscher Wetterdienst) Warnings displayed using MeteoAlarm.

would rely on expert knowledge rather than quantitative data. However, the range of expert knowledge required is broad; not exclusively from those with knowledge of the primary hazard but also from experts in other fields which may be affected through the secondary and tertiary hazards.

The actions to be taken will depend on the likelihood and severity of the scenario with a colour assigned based on an assessment of the risk (Fig. 1). Consequently, a disease outbreak or flood, which is highly likely to occur with severe impacts, is colour-coded red for high risk. The designation of the colours is subjective and dependent on combining sectorspecific knowledge. Historical or climatology-based regional and/or seasonal specific thresholds (in the case of meteorology or hydrology) can provide a valuable starting point for discussions in estimating the severity and the impact of an event.

In the case of flood risk, this may involve water resource managers, irrigation experts, and dam operators as well as disaster managers. The level of risk can be assigned to a specific geographical location - a grid box within the warning map - thereby building a dynamic risk map, which highlights the areas which may require specific interventions to mitigate the risk - house-to-house notifications, cell broadcasts, evacuation to shelters, etc. In particular, it will help civil protection to deploy their resources more effectively. Since the system is dynamic, this is a means to progressively express changing expectations of risk as a function of varying exposure, vulnerability and hydrometeorological likelihood. In the case of a tropical cyclone, for example, the flood risk would be identified based on the trajectory and intensity of the rainfall hazard within the event, among other factors. The risk matrix combines the flood and vulnerability information for each identified geographical section. As the tropical cyclone system evolves, the severity of the risk will change enabling an adaptive response to the event. Each of the risks associated with the secondary and tertiary hazards would also be estimated.

Unfortunately, risk data management is almost nonexistent in many countries and alternative approaches are needed to acquire the appropriate data.

\subsection{Advisories and Alerting}

Warning advisories and actions matrices are the final stage in the production process. These relate warnings and actions to the probability of an impact based on the impact risk matrix. Effective standard operating procedures are a critical component of the successful management of risk. Key elements are good communication among all of the relevant stakeholders and timely action. Having a common impact framework is also very useful for complex disasters and can make it easier for authorities to take early preparatory action to focus their resources, and to provide more targeted warning services. For example, the complex consequences of multiple hazards is exemplified by the situation in Vanuatu, the Solomon Islands and Fiji during April 2020, which faced the simultaneous crises of Tropical Cyclone Harold and the COVID-19 pandemic ${ }^{5}$.

It is important that each advisory and message contains the same information and detail across all media - no matter what format is applied. This is core to ensuring community confidence in the authority, authenticity, and security of the messages they get. The Common Alerting Protocol (CAP) provides a format designed for any and all media to communicate information about any kind of hazard situation. It was developed to standardize the technical format of an alerting message, regardless of content, in a manner such that the alert message is both human-readable and machine-readable. The message can be targeted to the public at large, to certain designated groups such as disaster managers or first responders, or to specific individuals as needed. The African Telecommunications Union has called for harmonized actions by

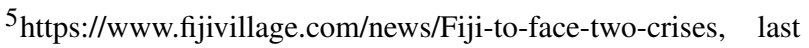
access: 26 June 2020
} 
telecommunication regulators by implementing $\mathrm{CAP}$ as one element of a strategy to combat COVID- $19^{6}$.

A message formatted with the CAP standard can be carried over or displayed by television, radio, mobile telephone, fax, highway signs, e-mail, the Web, etc. The message can communicate about weather, fires, earthquakes, volcanoes, landslides, child abductions, disease outbreaks, air quality warnings, transportation problems, power outages, etc., and can be fully integrated with the Meteoalarm system. While the technical specifications of CAP follow an international standard, CAP messaging can be modified and adapted to suit national requirements.

Without CAP, alerting systems must deal with free text or with different formats that vary across hazard types, and across countries as well. Without a standard alert format, it is practically impossible to implement all-hazard, allmedia public alerting (WMO, 2013). With the development of the CAP standard effective and efficient alerting systems promise great benefits for relatively modest investments. Finally, alerting authorities can use fairly simple tools to get critical messages to affected people, wherever they are and whatever they are doing.

A warning and alerting service is successful when recipients: receive the warning, via both formal and informal communication networks; understand the information presented; trust and believe the content, context and message; personalize the information; are enabled to make correct decisions; respond in an adequate and timely manner; and provide feedback and lessons learned. Among these factors understanding, trusting, believing and personalizing the information are extremely important leading to appropriate behaviour, correct decision making, and effective response by recipients (Anderson-Berry et al., 2018). Ineffective prior planning and preparation, haphazard and ad-hoc coordination among authorities to whom the population turns in times of crises, and the inability of authorities to articulate a uniform message, can lead to lack of trust, confusion and inability or unwillingness to act by the public. This is exacerbated if warning language is too complicated, vague, ambiguous or threatening, or is contradictory as can happen if it originates from different sources.

A primary factor that prompts people to trust and act on advice contained in warnings and alerts is the credibility of the issuing authority. People often judge and trust authorities on their expert skill, their past performance, and, on occasion, their freedom from political agendas and interference. This has been demonstrated variously across many nations in the COVID-19 pandemic and the reliance on information directly from health experts and authorities. Without trust and respect for authorities it becomes difficult to convince a population that the authorities have their best interest at heart and that whatever instructions are being issued are ultimately for

\footnotetext{
${ }^{6} \mathrm{https}: / / \mathrm{www}$. africanews.com/2020/03/23, last access: 26 June 2020
}

their well-being. A MHIEWS, which performs well in the cases of relatively frequent, low level warnings (as is the case for most meteorological hazards) is perceived by the public as a reliable source of information. Therefore, the system is a trusted and reliable source of warnings for the rare, but extremely damaging events.

\section{Decision support}

MHIEWS alone are necessary, but not sufficient tools in making effective decisions. Disaster management for all types of hazards must cover three broadly overlapping areas: - (1) Action in real-time to an immediate emergency situation, recovery and rehabilitation planning and implementation; (2) Early action planning and activation, triggered by impact based warnings of a threat with as much lead time as possible to effectively reduce or eliminate the risk to life, livelihoods and property, and to prepare for effective response; and (3) Long term planning and action to permanently eliminate or significantly reduce risks and to enhance effective preparedness.

Each of these three areas has a distinct and unique focus. Long-term Emergency Planning must have a primary goal of building resilience, capability and capacity to respond in emergency management authorities and at-risk communities and societies. It involves the establishment of robust protocols between Disaster Management at all levels and the relevant technical, societal and community welfare agencies and authorities for the reception of warnings related to hazards, processes to communicate these warnings effectively to at-risk communities, and the organisation of routine exercises to ensure smooth operation of the warning system. Emergency response requires situational awareness and the tools to make real-time decisions, which reduce the exposure of those at risk through evacuation, sheltering, rescue, self-rescue or the elimination of the threat if technological in origin. Anticipatory action implies advanced notification of a hazard and awareness of its potential impact. Lead-time, understanding, acceptance of the threat, the ability and willingness to take action, are all among the factors which play a role in the effective reaction to a warning where the aim is to reduce the potential for a full-scale emergency situation and need for a disaster response. Underlying each of these areas is knowledge of hazards, vulnerability and exposure, which also contribute to long-term planning to reduce vulnerability and thereby reduce the risk of an adverse impact.

Understanding the differentiated responsibilities of each of the actors involved in all three areas is essential for supporting the safety of lives and promoting the economic security of any country. Decision Support Systems (DSS) must provide guidance to all three areas. Information exchange and sharing among agencies is critical to the utility of a DSS and clear operating procedures are needed to ensure unimpeded information flows enabling hazard, vulnerability and expo- 
sure data to be combined with other guidance to promote timely action. The United Nations Office for Disaster Risk Reduction (UNDRR) ${ }^{7}$ provides guidance at national and regional levels on how government agencies from different sectors should work together to cover early warning, disaster response and recovery and long-term planning to mitigate the impact of disasters. A key module of a Disaster Management DSS is the "Resources for Response" at various levels. In many instances, the information on resources required to respond to a health emergency are not collected and included in such databases. While the response to a health emergency could benefit from the available data/information as part of a DSS, during the design and development of DSS, the resources needed to respond to a health emergency must be included.

Knowing who to warn requires knowledge of the hazards; knowing where to focus attention on refining the hazard warnings requires knowledge of the vulnerability and level of impacts. Consequently, all actors need to share data and information and collaborate closely to generate targeted and actionable information. Vulnerable disadvantaged and marginalised groups are often overlooked in the decisionmaking process. These include people with a range of physical, mental, social and psychological disabilities, who need earlier warnings to take action, tourists with no knowledge of local hazards and risks, minority populations, migrant workers or refugees with limited access to local information systems or the knowledge of the local language (UNDRR, 2020). It is extremely important to have an adequate information system that would include information on vulnerable groups as they are among those most at risk during any kind of hazardous events and the decision makers who can activate early actions based on warnings. Decision making should take into account people who don't have access to information or to any mitigation tools, including financial tools, and who are not part of the decision-making process, as well as devising strategies to minimise the numbers in this cohort through awareness building campaigns.

A DSS must encompass, therefore, providing for the internal information requirements of all government actors, as well as generating the guidance needed by non-governmental decision-makers and the general public. The basic building blocks of a DSS are based on a MHIEWS, which includes communication modules and a common data repository allowing multiple users to derive products relevant to their own requirements and to enhance the overall functionality of the DSS. This would comprise vulnerability and exposure information and data on primary, secondary, and tertiary hazards and impacts; an alerting module based on impact-based warnings; an external communication module, which facilitates the onward promulgation and exchange of information with the public or affected sectors or both; the means to share

\footnotetext{
${ }^{7}$ https://www.undrr.org/implementing-sendai-framework/ regional-platforms, last access: 26 June 2020
}

information with other systems supporting emergency operations (including the media and emergency planning); and clear operating procedures adhered to by all stakeholders.

Essential to the system is consistency and trust. Warning services must be consistent and must engender trust in order for people to take appropriate action (Hua and Shaw, 2020). The messages must be understood and actionable. This requires a common understanding of threat level across all hazards and impacts. Increasingly it also means that people need to understand that a low likelihood of medium and high impact events may also require a response from all those potentially affected. This requires a strategy for frequent exercises based on realistic scenarios, which should also be an element of the DSS since it will increase knowledge of vulnerability. In turn, this will help improve the utility of impact-based warnings. It is also very important after each event to review the performance of the system and gain feedback from users to further improve the system.

Generally, hazard impacts are localized. The DSS must therefore support decision-making at all levels of government (national, regional, local) with clear operating procedures in place to facilitate sound decisions at each level and between all agencies. The structure and flow of information should aim at making emergency response unnecessary by encouraging and guiding appropriate behaviour of the people affected. In many circumstances, the notion of self-rescue applies - meaning those impacted will have the capacity to take appropriate actions to reduce adverse risk with minimal or no intervention from authorities. This is an important point. If a warning is issued with a decent lead time, a lot of people who have options with friends and family will self-evacuate. This will reduce the cost to the Government significantly and will allow the Government to accommodate the most vulnerable people, who do not have such options, in shelters. Again, consistency and trust of the warning information is critically important in facilitating appropriate individual and community actions, together with an empowerment of citizens through the provision of clear and actionable guidance.

\section{Conclusions}

The ability to understand and respond effectively to warnings is central to resilient societies and communities. By avoiding physical, societal and economic harm to the greatest extent possible, recovery from a hazard is likely to be faster, less costly and more complete (Rogers et al., 2018). Meteorological Services have demonstrated that impact-based forecast and warning services based on the fundamental elements of a well-trusted, people-centred warning system can complement traditional warning systems and services by translating technical knowledge into actionable information of direct relevance to those affected. The use of probabilistic techniques gives us insight into the likelihood of a hazard, and we can use this knowledge, coupled with information about what 
and who is likely to be affected, to provide more actionable warnings (Rogers et al., 2019).

The MHIEWS paradigm can become a common approach for all hazards and therefore more likely to be a tool that everyone can understand and use as a basic element in their national disaster management system. The interconnectedness of hazards is a strong motivator for a common approach, especially where rapidly changing weather patterns increase flood, heat and epidemic risks (Ghebreyesus et al., 2008; Liu et al., 2014, 2020). One of the lessons from the COVID-19 pandemic is the need to provide reliable warnings and to be willing to prepare for a reasonable worst-case scenario based on informed long-term planning. Meteorology and hydrology are making progress in this direction and the processes developed in these disciplines can be readily applied to health and other sectors.

Effective multi-hazard impact-based early warning systems are based on authoritative and timely risk information. Creation of this information requires systematic and standardized processes to collect, assess and share data concerning exposure, hazards and vulnerabilities. Risk must be recognised as being dynamic as vulnerability, the nature of hazards, and the extent of exposure change over time due to many factors, including urbanization, rural land-use change, environmental degradation and climate change. It is extremely important to make sure that vulnerable groups and communities are included in the processes of risk assessment and communication. It is equally important to make decisions that protect vulnerable groups taking into account the realities that people find themselves in - often without access to information or tools to mitigate the damage, including financial tools.

For the poorest countries, many of which know only too well the extent of the damage natural hazards can lead to, the full danger of COVID19 is only just coming into view ${ }^{8}$. Similarly, vulnerable communities are challenged by fragile health system, loss of critical income, lack of medical supply or financial support. MHIEWS should be a tool that works in the interest of these groups and that guides governments' decisions that - whether they are related to re-locating people or to restricting their movements - do not create any counter incentives and provide "shelter" in more than just one sense of the word.

As COVID-19 seems likely to be endemic with no vaccine or other mitigating measures on the immediate horizon (Nabarro and Colombano, 2020), a CAP-based warning system is essential if we are to alert people to potential threats as we learn to live with it. In the long term, a comprehensive multi-hazard impact-based early warning system is needed to prepare us for all potential threats from natural and other

\footnotetext{
${ }^{8}$ https://blogs.worldbank.org/voices/ poorest-countries-full-danger-coronavirus-only-just-coming-view? CID=WBW_AL_BlogNotification_EN_EXT, last access: 26 June 2020
}

hazards in a systematic way. As a potential starting point, the MHIEWS could focus on heat health, for which there is a growing body of knowledge on the impacts of both high and low temperatures on excess mortality (Matthies et al., 2008; Liu et al., 2014). Air quality and health is another area for cooperation. Recent studies indicate a spatial correlation between ambient particulate matter (PM) pollution and increase COVID-19 related death rates (Yao et al., 2020a; Andrée, 2020). This would also link the longer-term consequences of climate change with the immediate needs of society to cope with rising temperatures, extreme cold and air quality, while also generalizing the tools to support the detection and early warning of infectious disease epidemics and pandemics, which may or may not be related to climate (Yao et al. 2020b).

COVID-19 is creating a new reality, beyond the immediate health impacts, by pushing 40-60 million people into extreme poverty ${ }^{9}$ increasing the vulnerability of people to the impact of other hazards. Reinforcing the use of impact-based forecasting and warning services with a common framework of anticipatory action will help to minimize future disasters.

Data availability. No data sets were used in this article.

Author contributions. DPR prepared the manuscript with contributions from all co-authors.

Competing interests. The authors declare that they have no conflict of interest.

Special issue statement. This article is part of the special issue "19th EMS Annual Meeting: European Conference for Applied Meteorology and Climatology 2019". It is a result of the EMS Annual Meeting: European Conference for Applied Meteorology and Climatology 2019, Lyngby, Denmark, 9-13 September 2019.

Acknowledgements. The authors would like to thank Abbas Kumar Jha, Stephan Hallegatte, Ana Campos Garcia, and Michel Jancloes for their help in improving the paper.

Financial support. This work was supported by the World Bank and the Global Facility for Disaster Risk Reduction (GFDRR).

Review statement. This paper was edited by Tanja Cegnar and reviewed by Will Lang and Catalina Jaime.

\footnotetext{
${ }^{9}$ https://blogs.worldbank.org/opendata/, last access: 26 June 2020
} 


\section{References}

Anderson-Berry, L., Achilles, T., Panchuk, S., Mackie, B., Canterford, S., Leck, A., and Bird, D. K.: Sending a message: How significant events have influenced the warnings landscape in Australia, Int. J. Disast. Risk Re., 30, 5-17, https://doi.org/10.1016/j.ijdrr.2018.03.005, 2018.

Andrée, B. P. J.: Incidence of COVID-19 and Connections with Air Pollution Exposure: Evidence from the Netherlands, World Bank, Paris, France, 2020.

Cho, H., Ippolito, D., and Yu, Y. W.: Contact tracing mobile apps for COVID-19: privacy considerations and related trade-offs. ArXiv, [preprint], arXiv:2003.11511v2, 2020.

Chowell, G., Luo, R., Sun, K., Roosa, K., Tariq, A., and Viboud, C.: Real-time forecasting of epidemic trajectories using computational dynamic ensembles, Epidemics, 30, 100379, https://doi.org/10.1016/j.epidem.2019.100379, 2020.

Coughlan de Perez, E., van den Hurk, B., van Aalst, M. K., Jongman, B., Klose, T., and Suarez, P.: Forecast-based financing: an approach for catalyzing humanitarian action based on extreme weather and climate forecasts, Nat. Hazards Earth Syst. Sci., 15, 895-904, https://doi.org/10.5194/nhess-15-895-2015, 2015.

Ghebreyesus, T. A., Tadese, Z., Jima, D., Bekele, E., Mihretie, A., Yihdego, Y. Y., Dinku, T., Connor, S. J., and Rogers, D. P.: Public health and weather services - climate information for the health sector, WMO Bulletin, 57, 256-261, 2008.

Gros, C., Bailey, M., Schwager, S., Hassan, A., Zingg, R., Uddin, M. M., Shahjajan, M., Islam, H., Lux, S., Jaime, C., and de Perez, E. C.: Household-level effects of providing forecast-based cash in anticipation of extreme weather events: Quasi-experimental evidence from humanitarian interventions in the 2017 floods in Bangladesh, Int. J. Disast. Risk Re., 41, 1-11, 2019.

Harford, T.: Why we fail to prepare for disasters, Financial Times Magazine, 2020.

Hua, J. and Shaw, R.: Corona Virus (COVID-19) "Infodemic" and emerging Issues through a data lens: The case of China, Int. J. Environ. Res. Pub. He., 17, 2309-2321, 2020.

IFRC: Community early warning systems: guiding principles, International Federation of the Red Cross and Red Crescent Societies, 82 pp., 2012.

Kahneman, D.: Thing, Fast and Slow, Farrar, Straus and Giroux, New York, 2011.

Kluge, H. H. P.: Statement - Countries must work together as COVID-19 pandemic accelerates. World Health Organization - Regional Office for Europe, available at: http://www.euro.who.int/en/health-topics/healthemergencies/coronavirus-covid-19/statements/statementcountries-must-work-together-as-covid-19-pandemicaccelerates, last access: 26 June 2020, 2020.

Liu, Q., Tan, Z.-M., Sun, J., Hou, Y., Fu, C., and Wu, Z.: Changing rapid weather variability increases influenza epidemic risk in a warming climate, Environ. Res. Lett., 15, 044004, https://doi.org/10.1088/1748-9326/ab70bc, 2020.

Liu, Y., Kan, K., Xu, J., Rogers, D., Peng, L., Ye, X., Chen, R., Zhang, Y., and Wang, W.: Temporal relationship between hospital admissions for pneumonia and weather conditions in Shanghai, China: a time series analysis, BMJ Open, 4, e004961, https://doi.org/10.1136/bmjopen-2014-004961, 2014.
Matthies, F., Bickler, G., Marin, N. C., and Hales, S.: Health-health action plans: Guidance, WHO Regional Office for Europe, 2008.

Moteff, J. D.: Critical Infrastructure Resilience: The evolution of policy and programs and issues for Congress. Congressional Research Service, 7-5700 Washington DC., 20 pp., available at: http://fas.org/sgp/crs/homesec/R42683.pdf, last access: 26 June 2020, 2012.

Murphy, A. H.: On expected-utility measures in cost-loss ratio decision situations, J. Appl. Meteorol., 8, 989-991, 1969.

Nabarro, D. and Colombano, J.: About COVID-ready economies, Covid-19 Narratives, Narrative eighteen 10 April, 2020.

Palmer, T.: The ECMWF ensemble prediction system: Looking back (more than) 5 years and projecting forward 25 Years, Q. J. R. Meteor. Soc., 145, 12-24, 2019.

Rogers, D. P. and Tsirkunov, V. V.: Weather and Climate Resilience: Effective Preparedness through National Meteorological and Hydrological Services, Directions in Development, Washington, DC: World Bank, 2013.

Rogers, D. P., Kootval, H., and Tsirkunov, V. V.: Early Warning, Resilient Infrastructure and Risk Transfer, in World Scientific Series on Asia-Pacific Weather and Climate: Bridging Science and Policy Implication for Managing Climate Extremes, edited by: Jung, H.-S. and Wang, B., World Scientific Series on AsiaPacific Weather and Climate, 10, 65-79, 2018.

Rogers, D. P., Tsirkunov, V. V., Kootval, H., Soares, A., Kull, D. W., Bogdanova, A.-M., and Suwa, M.: Weathering the Change: How to Improve Hydromet Services in Developing Countries, World Bank, Washington, D.C., 2019.

Staudinger, M.: The EMMA/METEOALARM Multiservice Meteorological Awareness System, WMO/TD-No 1438, Geneva, 2008.

Taleb, N. N.: The Black Swan: the impact of the highly improbable, Second Edition, Random House, 2010.

Tang, X., Feng, L., Zou, Y., and Mu, H.: The Shanghai Multi-hazard Warning System: Addressing the Challenge of Disaster Risk Reduction in an Urban Megalopolis, in: Institutional Partnerships in Multi-Hazard Early Warning Systems, edited by: Golnaraghi, M., Heidelberg, Germany, Springer, 159-179, 2012.

UNDRR: Leave no one behind in COVID-19 prevention, response, and recovery, United Nations Disaster Reduction and Recovery Asia Pacific COVID-19 Brief, 2020.

United Nations: Sendai Framework for Disaster Risk Reduction 2015-2030, United Nations, 2015.

Webster, P. J., Jian, J., Hopson, T. M., Hoyos, C. D., Agudelo, P. A., Chang, H.-R., Curry, J. A., Grossman, R. L., Palmer, T. N., and Subbiah, A. R.: Extended-range probabilistic forecasts of Ganges and Brahmaputra floods in Bangladesh, B. Am. Meteorol. Soc., 91, 1493-1514, https://doi.org/10.1175/2010BAMS2911.1, 2010.

WMO: Guidelines for Implementation of Common Alerting Protocol (CAP)-enabled emergency alerting. World Meteorological Organization TD 1109, 2013.

WMO: WMO guidelines on multi-hazard impact-based forecast and warning services, World Meteorological Organization TD no. $1150,2015$.

WMO: The Second Multi-Hazard Earl warning Conference (MHEWC-II). 13-14th May, World Meteorological Organization, Geneva, Switzerland, available at: https://mhews.wmo.int/ en/welcome, last access: 26 June 2020, 2019. 
Yao, Y., Pan, J., Wang W., Liu, Z., Kan, H., Meng, X., Wang, W.: Spatial Correlation of Particulate Matter Pollution and Death rate of COVID-19, MedRxiv, [preprint], https://doi.org/10.1101/2020.04.07.20052142, 2020a.
Yao, Y., Pan, J., Liu, Z., Meng, X., Wang, W., Kan, K., and Wang, W.: No association of COVID-19 transmission with temperature or UV radiation in Chinese cities, Eur. Respir. J., 55, 2000517, https://doi.org/10.1183/13993003.00517-2020, 2020b. 